\title{
PRIMER REGISTRO DE LAMPRIS GUTTATUS (PISCES: LAMPRIDIDAE) EN EL GOLFO DE ARAUCO, VIII REGION, CHILE
}

\section{FIRST RECORD OF LAMPRIS GUTTATUS (PISCES: LAMPRIDIDAE) IN THE GULF OF ARAUCO, VIII REGION, CHILE}

\author{
Víctor H. Ruiz R. ${ }^{1} \&$ Ricardo Figueroa J. ${ }^{2}$ \\ ${ }^{1}$ Universidad de Concepción, Departamento de Zoología, Casilla 160-C Concepción, Chile; Email: vruiz@udec.cl \\ ${ }^{2}$ Unidad de Sistemas Acuáticos, Centro EULA-Chile, Universidad de Concepción, Casilla 156-C, Concepción, Chile. \\ Email: rfiguero@udec.cl
}

\section{RESUMEN}

Se registra por primera vez para el Golfo de Arauco la presencia del pez “opah” Lampris guttatus (Brunnich,1788), sobre la base de la captura de dos ejemplares, durante los meses de agosto de 1991 y septiembre de 2001. El ejemplar de mayor tamaño (1360 mm de longitud total y 73 kilos) se encuentra depositado en la colección ictiológica del Museo Zoológico de la Universidad de Concepción (MZUC/UCCC), el segundo ejemplar mide 1.290 mm y alcanzó un peso de $70 \mathrm{~kg}$. El análisis del contenido estomacal reveló la presencia de restos digeridos de peces, moluscos y crustáceos. Un intestino relativamente corto confirma su régimen de alimentación carnívora.

Palabras claves: Nuevo registro, Lamprididae, Lampris, Golfo de Arauco, Chile.

\begin{abstract}
Lampris guttatus (Brunnich, 1788) "opah" have been recorded in the Gulf of Arauco (Chile) after the captures of two specimens (August 1991 and September 2001). The first specimen weighed $73 \mathrm{~kg}$ with a total length of $1360 \mathrm{~mm}$, the second, $70 \mathrm{~kg}$ and $1290 \mathrm{~mm}$. The largest and heaviest specimen is deposited in the ictiological collection of the Zoological Museum of the University of Concepcion (MZUC/UCCC). The analysis of the stomach content revealed the presence of digested fishes, molluscs and crustaceans. Its relatively short intestine confirms its carnivorous diet.
\end{abstract}

Keywords: New record, Lamprididae, Lampris, Gulf of Arauco, Chile.

\section{INTRODUCCION}

El orden Lampridiformes se encuentra representado en Chile por las familias Lamprididae (Lampris regius (Bonnaterre 1788)), Regalecidae (Regalecus glesne Ascanius, 1772), Stylephoridae (Stylephorus chordatus Shaw, 1791) y Trachipteridae (Desmodema polystica (Ogilby, 1897); Trachipterus altivelis (Ogilby 1897); Trachipterus fukusakii Fitch, 1964; Trachipterus trachipterus (Gmelin 1789); y Zu cristatus (Bonnellí 1820)) (Pequeño 1989, 1997). La familia Lamprididae incluye dos especies oceánicas y epipelágicas: Lampris guttatus (Brunnich 1788) de distribución casi cosmopolita (Fowler 1928: 89, Jordan et al. 1930: 216, Herald 1939: 228, Fitch 1951: 97, Gosline \& Brock 1965:145-1, Fitch \& Lavenberg 1968: 84, Parin 1968: 54, Leim \& Scott 1972: 248, Millar \& Lea 1972: 84, Hart 1973: 269, Palmer 1973: 328, Guitart 1975: 287, Eschmeyer et al. 1983, Parin \& Kukuyev 1983: 8, Heemstra 1986: 398, Palmer 1986: 725, Gon 1990: 215, McEachran \& Fechhelm 1998: 682 fide Castro-Aguirre et al. 2001) y L. inmaculatus Gilchrist, 1904, especies que son reconocidas 
taxonómicamente por Parin \& Kukuyev (loc. cit) para el océano del sur, entre $45^{\circ} \mathrm{S}$ y $50^{\circ} \mathrm{S}$ (fide Castro-Aguirre et al. 2001). La especie L. guttatus ha sido registrada en varias oportunidades para la costa de California. Herald (1939) señala la presencia de 24 ejemplares, la mayoría, sin embargo, de tamaño un poco inferior a los reportados aquí. Fitch (1951) reporta cinco ejemplares y habla de una distribución a lo largo de la costa del Pacífico entre el Sur de California y Alaska. Castro-Aguirre et al. (2001) entregan un segundo registro de L. guttatus para la costa del suroeste del Golfo de California. No se dispone de literatura que haya reportado la especie L. guttatus (opah, luna real o pez sol) para el Golfo de Arauco, es más, no existen antecedentes a disposición que lo citen para Chile, aunque aparece en la lista de peces de Pequeño (1989) como $L$. regius (Bonnaterre 1788). De manera que éste podría ser también el primer registro de la especie para Chile, al no estar documentada para un área específica del país. El cambio nomenclatural de regius a guttatus fue documentado por Palmer \& Oelschläger (1976) pasando regius a ser sinónimo de guttatus. Este registro se hace sobre la base de la captura de dos ejemplares, uno recolectado en agosto de 1991 en el Golfo de Arauco (Concepción, Chile) que correspondió a un macho adulto de 1360 mm de longitud total y 73 kilogramos de peso (Fig. 1, Tabla I). El espécimen se encontraba nadando lentamente y con parte del cuerpo asomado a la superficie. El segundo fue recolectado en la misma zona (septiembre de 2001) durante las capturas de clupeidos y fauna acompañante mediante una red de cerco aproximadamente a 30-50 metros de profundidad. No fue posible determinar el sexo porque no se observó evidencia de gónada, pero era de menor tamaño (1290 mm de longitud total y 70 kilos) que el anterior (Tabla I).

La disección de los especímenes permitió observar gran desarrollo de los músculos y huesos en relación con la cintura pectoral. El corazón, estómago e hígado eran de tamaño considerable, lo que coincide con lo reportado por Rosenblatt \& Johnson (1976). El estómago mostraba restos de algunos peces, calamares, pulpos y crustáceos posiblemente isópodos. Un intestino relativamente corto confirma que el pez es de hábitos carnívoros. No se encontraron parásitos externos ni internos. La carne era de color rosado, pero por desconocimiento no fue consumida y se desechó.

TABLA I. Valores de algunos caracteres morfométricos y merísticos de los especímenes del Golfo de Arauco comparados con el ejemplar de Castro-Aguirre et al. 2001 del Golfo de California.

TABLE I. Comparative values of morphometric and meristic character of two specimens from Gulf of Arauco (this work) and one from Gulf of California (Castro-Aguirre et al. 2001**).

\begin{tabular}{lccc}
\hline Caracteres considerados $(\mathrm{mm}) *$ Ejemplar 1 & \multicolumn{2}{c}{ Ejemplar 2 } & Ejemplar 3** \\
& $(73 \mathrm{~kg}).(70 \mathrm{~kg}).(35 \mathrm{~kg})$. & \\
\hline Longitud total & 1360 & 1290 & 1050 \\
Longitud estándar & 1150 & 1090 & 985 \\
Altura máxima del cuerpo & 740 & 650 & $292($ Sic!) \\
Altura mínima pedúnculo caudal & 85 & 80 & - \\
Longitud de la cabeza & 402 & 385 & 363 \\
Altura de la cabeza & 420 & 405 & 400 \\
Diámetro del ojo & 80 & 70 & 640 (Sic!) \\
Longitud de la aleta pectoral & 290 & 290 & 292 \\
Longitud de la aleta pélvica & 245 & 250 & 260 \\
Número de radios de la dorsal & 50 & 53 & 53 \\
Número de radios de la anal & 42 & 40 & 39 \\
Número de radios de la pectoral & 23 & 23 & 23 \\
Número de radios de la pélvica & 14 & 14 & 14 \\
Número de branquispinas & 14 & 14 & 14 \\
\hline
\end{tabular}

* La unidad de medida es válida sólo para los caracteres morfométricos y no para los merísticos (número de radios y branquispinas). 


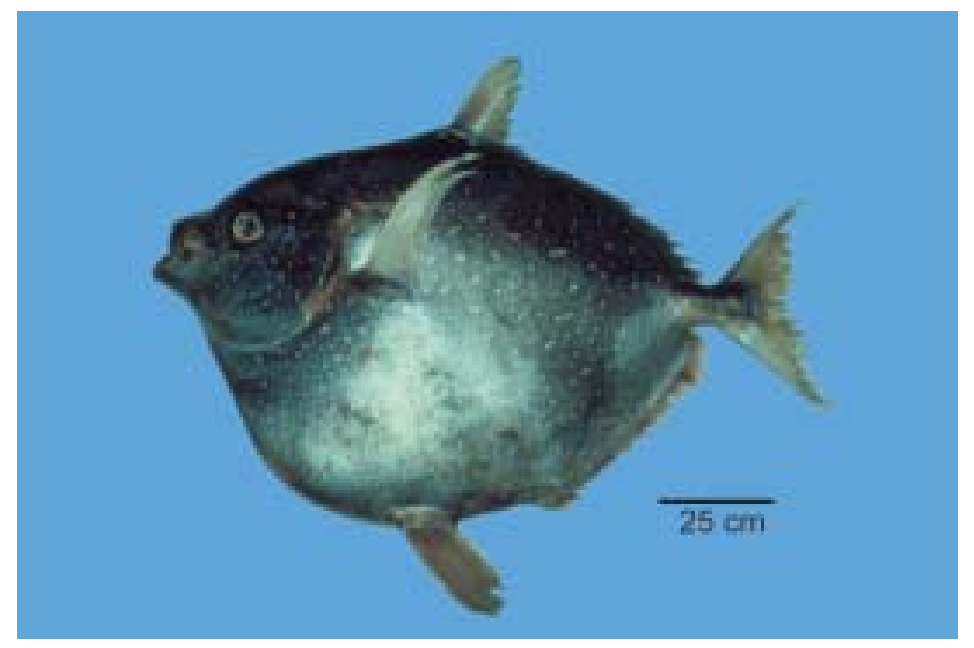

FiguRA 1. Ejemplar de Lampris guttatus (1360 mm LT) recolectado en agosto de 1991 en el Golfo de Arauco, Chile.

FIGURE 1. Specimen of Lampris guttatus (1360 mm TL) found on August, 1991 in Gulf of Arauco, Chile.

El ejemplar de mayor tamaño se encuentra preservado en la colección ictiológica de la Universidad de Concepción bajo el No 22178-MZUC/ UCCC.

Finalmente, podemos concluir que la especie en el país y sobre todo en el área de la Octava Región puede ser mucho más abundante ya que existen comunicaciones personales de pescadores que dicen haberlo capturado y consumido frecuentemente.

\section{AGRADECIMIENTOS}

Se agradece al Sr. Enrique Conejeros R., quien donó los especímenes al Departamento de Zoología de la Universidad de Concepción, y al Sr. Ernesto Toledo L. por la preservación de uno de los ejemplares y su ayuda en la disección de los mismos. A Hugo Iván Moyano y Margarita Marchant por la lectura crítica del manuscrito, y también a dos revisores anónimos.

\section{BIBLIOGRAFIA}

Castro-Aguirre, J. L., G. de la Cruz-Agüero, J. de la Cruz-Agüero \& A. F. GonzÁlez-Acosta. 2001. A second record of Lampris guttatus (Pisces: Lamprididae) from the southwestern coast of the Golfo de California, México. Oceanides 16(2): 139-141.

Fiтch, J. E. 1951. Studies and notes on some California marine fishes. Calif. Fish \& Game 37(2): 111-120.

Herald, E.S. 1939. The opah (Lampris regius) and its occurrence off the California coast. Calif. Fish Game 25(3): 228-232.

Palmer, G. \& H.A. Oelschläger. 1976. Use of the name Lampris guttatus (Brünnich, 1788) in preference to Lampris regius (Bonnaterre, 1788) for the opah. Copeia 1976(2): 366-367.

Pequeño, G. 1989. Peces de Chile. Lista Sistemática revisada y comentada. Rev. Biol. Mar., Valparaíso 24(2): 1-132.

Pequeño, G. 1997. Peces de Chile. Lista Sistemática revisada y comentada: Addendum. Rev. Biol. Mar. y Oceanografía 32 (2): 77-94.

Rosenblatt, R.H. \& G.D. Johnson. 1976. Anatomical considerations of pectoral swimming in the opah, Lampris guttatus. Copeia 1976(2): 367-370.

Fecha de recepción: 06.12.05

Fecha de aceptación: 05.01.06 